\title{
Abdominal Wall Closure in Autologous Breast Reconstruction: Optimizing Aesthetics
}

\author{
Peter Deptula, Dung Nguyen* \\ Division of Plastic and Reconstructive Surgery, Stanford University Medical Center, Stanford, California, USA
}

\section{Email address:}

nguyendh@stanford.edu (D. Nguyen)

${ }^{*}$ Corresponding author

\section{To cite this article:}

Peter Deptula, Dung Nguyen. Abdominal Wall Closure in Autologous Breast Reconstruction: Optimizing Aesthetics. Journal of Surgery. Vol. 9, No. 3, 2021, pp. 128-133. doi: 10.11648/j.js.20210903.16

Received: April 25, 2021; Accepted: May 7, 2021; Published: May 27, 2021

\begin{abstract}
Background: In contrast to cosmetic abdominoplasty, abdominal flap harvest can result a high degree of morbidity to the abdominal wall. Poor abdominal wall aesthetics that can result following free flap harvest including a high abdominal incision and post-operative hernia or bulge. We report our experience in optimizing cosmesis of the abdominal donor site with the use of a low incision, fascial plication and routine mesh reinforcement. Methods: A retrospective chart review of patients who underwent breast reconstruction with free abdominal tissue transfer from 2013-2017. Pedicled flaps and superior inferior epigastric artery flaps were excluded. Patient demographics, oncologic history, ablative and reconstructive surgery details focusing on abdominal closure techniques, and postoperative complications were evaluated. Results: 135 patients were identified who underwent 223 abdominal free flaps. 59 (26.5\%) DIEP flaps were harvested, while 160 (71.7\%) msTRAM and $4(1.8 \%)$ fTRAM flaps were harvested $(\mathrm{p}<0.0001) .160(71.7 \%)$ donor sites closures utilized polypropylene mesh, $9(4.0 \%)$ were closed with biologic mesh and $3(1.3 \%)$ closures used no mesh $(\mathrm{p}<0.0001)$. No hernias were observed $(0 \%)$, while 6 abdominal bulges were identified (2.7\%). There were no differences in the rates of abdominal bulge after donor site closure in the DIEP compared with msTRAM and fTRAM groups $(3.4 \%$ vs $2.4 \%, p=0.7)$. No patients required mesh explantation during the study follow up period. Conclusion: To parallel cosmetic abdominoplasty, our authors advocate for a low incision, fascial plication and routine mesh reinforcement of the abdominal wall following free flap harvest.
\end{abstract}

Keywords: Autologous Breast Reconstruction, Abdominal Wall, Abdominal Bulge, Abdominoplasty

\section{Introduction}

It is well known that patients undergoing autologous breast reconstruction with abdominal free tissue transfer demonstrate greater satisfaction and quality of life. [1] While much attention of patient outcomes has focused on the reconstructed breast, the secondary abdominal donor site has also emerged as a significant factor in patient outcomes. [2, 3] Studies evaluating patient reported outcomes have demonstrated superior patient well-being of the abdomen when muscle-preserving abdominally based flaps were utilized. $[1,4,5]$

In comparison to cosmetic abdominoplasty, abdominal flap harvest has traditionally prioritized the harvest of large periumbilical perforators. Here, incisions are placed higher on the abdominal wall resulting in a visible transverse abdominal scar. [2, 3, 6] Conversely, an abdominoplasty employs a discrete low-transverse incision. In addition, the presence of rectus diastasis and abdominal wall laxity is often treated with plication during cosmetic abdominoplasty, which is not routine in abdominal donor site closure.

Following harvest of an abdominal free flap, the abdominal wall is weakened, which can result in additional problems of hernia and bulge. [1] Bulging of the abdominal wall is attributed to fascial attenuation, muscle weakness or denervation, while a hernia represents a true defect in the abdominal wall. [7] The literature demonstrates rates of postoperative abdominal wall hernia and bulge up to $9 \%$ and $33 \%$, respectively. [4, 8-11] Muscle preservation techniques have resulted in improved abdominal wall function and a lower rate of complications. $[1,5]$

A multitude of techniques have been described to address the secondary abdominal donor site defect in autologous breast reconstruction. $[8,12]$ Our authors aim to establish an 
abdominal closure technique that parallels the aesthetics of a cosmetic abdominoplasty. We report our experience in optimizing cosmesis of the abdominal donor site with the use of a low incision, fascial plication and mesh reinforcement.

\section{Methods}

A retrospective chart review of patients who underwent immediate or delayed deep inferior epigastric artery perforator (DIEP) flap, muscle-sparing transverse rectus abdominis myocutaneous (msTRAM) flap or free transverse rectus abdominis myocutaneous (fTRAM) flap for breast reconstruction from 2013-2017. Pedicled flaps and superior inferior epigastric artery flaps were excluded. All procedures were performed by the senior author (DHN). Patient demographics, oncologic history, ablative and reconstructive surgery details focusing on abdominal closure techniques, and postoperative complications were evaluated. Unpaired $t$ tests and one-way ANOVA were used for data analysis. Pvalues less than 0.05 were deemed statistically significant.

Surgical Technique: Abdominal Donor-Site Closure

A low inferior transverse incision is prioritized which is designed $6-8 \mathrm{~cm}$ from the vulvar commissure. Abdominal free flaps (DIEP, msTRAM, and fTRAM) were harvested in standard fashion. The superior abdominal skin flap is elevated to the xiphoid and costal margins. In the case of msTRAM and fTRAM, the fascial defects are closed with an inlay mesh. Here, the mesh is sutured to the linea alba and linea semilunaris. The remaining anterior rectus fascia can then be primarily closed or approximated in the case of excessive tension. Figures 1 and 2 illustrate this closure technique. In the case of DIEP flap, the fascia is primarily closed without inlay mesh placement. In cases of rectus diastasis, fascial plication is performed centrally. To further improve on abdominal wall contour lateral plication is also performed. An onlay mesh is then placed over the anterior abdominal wall (Figure 3). Two $19 \mathrm{Fr}$ abdominal drains are then placed. The incision is then closed in a layered fashion at the level of Scarpa's fascia, deep dermis followed by a running subcuticular stitch. An abdominal binder is placed at the conclusion of surgery, which patients are instructed to wear for a total of 6 weeks post-operatively.
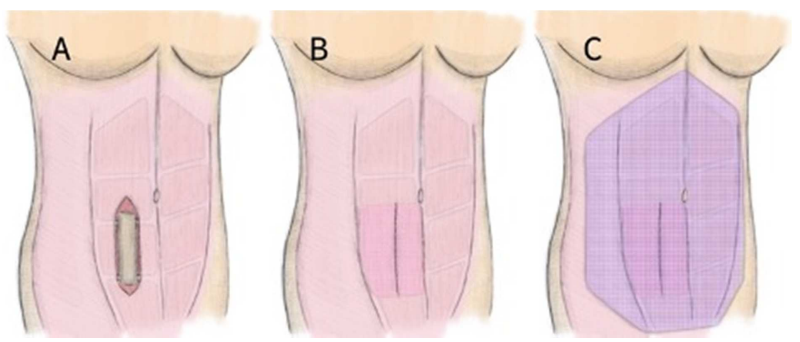

Figure 1. Mesh placement at MS-TRAM donor site. (A) The abdominal wall is displayed after MS-TRAM flap harvest. (B) An inlay mesh is placed at the $M S-T R A M$ donor site at fixed to the linea semilunaris and linea alba. The spared anterior recut sheath is closed primarily. (C) After inlay mesh placement and primary fascial closure, an overlay mesh is secured spanning the anterior abdominal wall.

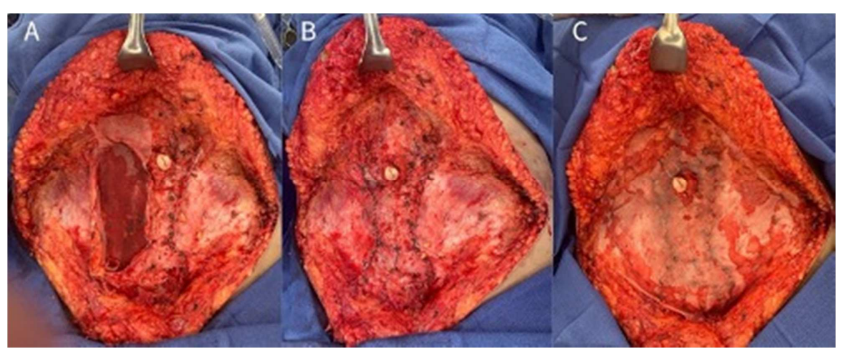

Figure 2. Intraoperative mesh placement at MS-TRAM donor site. (A) After MS-TRAM harvest, an inlay mesh is secured to the linea alba and linea semilunaris. (B) The preserved anterior rectus sheath is closed over the mesh inlay. (C) A spanning overlay mesh is placed along the anterior abdominal wall.
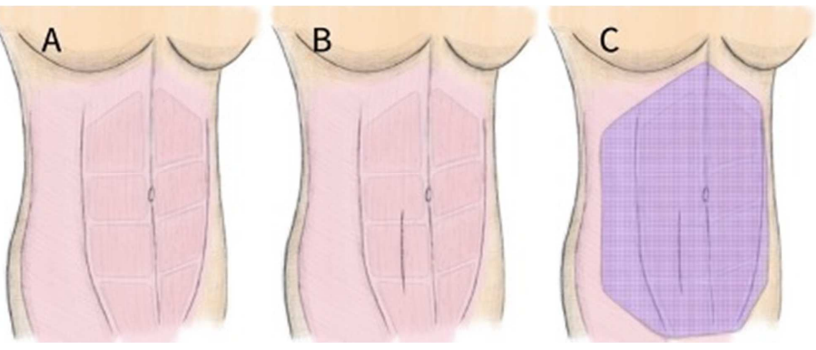

Figure 3. Mesh placement at DIEP donor site. (A) The abdominal wall is illustrated prior to DIEP flap harvest with fascia overlying the rectus abdominis muscle intact. (B) The anterior rectus sheath spared during DIEP flap harvest is closed primarily. (C) Polypropylene mesh is placed in an overlay fashion spanning the anterior abdominal wall.

\section{Results}

A total of 135 patients were identified whose data are summarized in Table 1. Patient age ranged from 26-72 years with BMI ranging from 17.9 to 42.3 . 6 patients $(4.4 \%)$ were active smokes, while $3(2.2 \%)$ patients had a diagnosis of diabetes mellitus. Prior abdominal surgery was noted in 33 patients $(24.4 \%)$. 25 patients carried a genetic mutation related to their oncologic surgery $(18.5 \%)$ : BRCA1 and BRCA 2 (15.6\%), VUS (1.3\%), CHEK2 (0.7\%), P53 (0.7\%). Indication for mastectomy was invasive carcinoma in 122 patients $(90.4 \%)$, while prophylactic in 13 patients $(9.6 \%)$. Chemotherapy was administered to $65(48.1 \%)$ patients and 45 patients underwent radiation therapy $(33.3 \%)$. All patients had a minimum of 6 months follow up time period with a range of 6.3 to 56 months following flap reconstruction.

223 abdominal free flaps that were performed as summarized by Table 2.138 flaps $(61.9 \%)$ were performed at the time of mastectomy and $85(38.1 \%)$ were performed in a delayed fashion $(\mathrm{p}<0.0001) .59$ (26.5\%) DIEP flaps were harvested, while $160(71.7 \%)$ msTRAM and 4 (1.8\%) fTRAM flaps were harvested $(\mathrm{p}<0.0001)$. Concurrent hernia repair at the time of flap surgery was performed in 7 patients $(5.2 \%)$.

$160(71.7 \%)$ donor sites closures utilized polypropylene mesh, $9(4.0 \%)$ were closed with biologic mesh and $3(1.3 \%)$ closures used no mesh $(\mathrm{p}<0.0001)$. With msTRAM and fTRAM harvest, all donor sites were closed with the use of mesh, as summarized in Table 3. Onlay mesh was used in 11 (6.7\%) flap closures, inlay in $12(7.3 \%)$, and a combination 
of inlay with onlay mesh in 141 closures (86.0\%). Polypropylene mesh was used in all 11 onlay repairs $(100.0 \%), 11$ inlay repairs $(91.7 \%)$ and 138 repairs with inlay and onlay mesh (97.9\%). Biologic mesh was used in $1(8.3 \%)$ inlay closure, while it was used in 3 closures as an inlay with onlay repair (2.1\%). After DIEP closure, mesh was used in 56 donor sites $(94.9 \%)$, the remaining 3 donor sites $(5.1 \%)$ were closed primarily without mesh reinforcement. Here mesh was exclusively placed in an onlay fashion. Polypropylene was used in 51 closures $(91.1 \%)$, while biologic mesh was used in 5 closures $(8.9 \%)$.

Table 1. Patient Characteristics.

\begin{tabular}{ll}
\hline Patient characteristics (n=135) & \\
\hline Age (years) & $26-72$ (range) \\
BMI $\left(\mathrm{kg} / \mathrm{m}^{2}\right)$ & $17.9-42.3$ (range) \\
Active smoker & 6 \\
Diabetes & 3 \\
Genetic Mutation & $21(15.6 \%)$ \\
BRCA 1 or 2 & $2(1.3 \%)$ \\
VUS & $1(0.7 \%)$ \\
CHEK2 & $1(0.7 \%)$ \\
P53 & \\
Prior abdominal surgeries & $21(15.6 \%)$ \\
Cesarean section & $3(2.2 \%)$ \\
Laparoscopic cholecystectomy & $3(2.2 \%)$ \\
Gastric Bypass & $6(4.4 \%)$ \\
Other* & \\
Indication & $13(9.6 \%)$ \\
Prophylactic & $122(90.4 \%)$ \\
Invasive carcinoma & \\
Chemotherapy & $29(21.5 \%)$ \\
Neoadjuvant & $36(26.7 \%)$ \\
Adjuvant & \\
Radiation & $11(8.1 \%)$ \\
Neoadjuvant & $34(25.2 \%)$ \\
Adjuvant & $6.3-56(\mathrm{range})$ \\
Follow-up time (months) &
\end{tabular}

Table 2. Free flap characteristics.

\begin{tabular}{lll}
\hline Free flap characteristics & Number of flapsn=223 (\%) & p-value \\
\hline Timing of Reconstruction & & \\
Immediate & $138(61.9 \%)$ & $<0.0001$ \\
Delayed & $85(38.1 \%)$ & \\
Type of Abdominal Free Flap & $<0.0001$ \\
msTRAM & $160(71.7 \%)$ & \\
fTRAM & $4(1.8 \%)$ & \\
DIEP & $59(26.5 \%)$ & \\
Concurrent hernia* & & \\
Umbilical hernia & $4(3.0 \%)$ & \\
Ventral hernia & $2(1.5 \%)$ & \\
Inguinal hernia & $1(0.7 \%)$ & \\
\hline
\end{tabular}

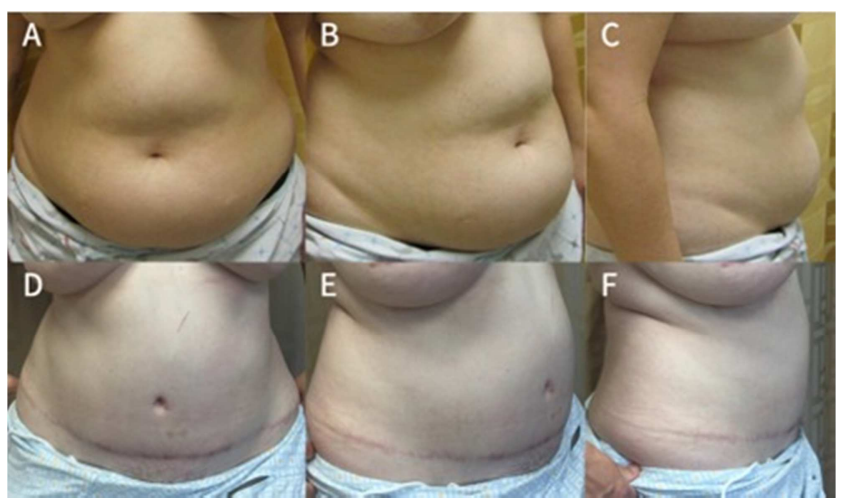

Figure 4. Patient 1 pre- and post-operative abdominal wall donor site. Example \#1 of abdominal wall donor site following msTRAM harvest using inlay and onlay polypropylene mesh. A-B: preoperative photographs. C-D: post-operative photographs demonstrating low incision and absence of hernia or bulge.

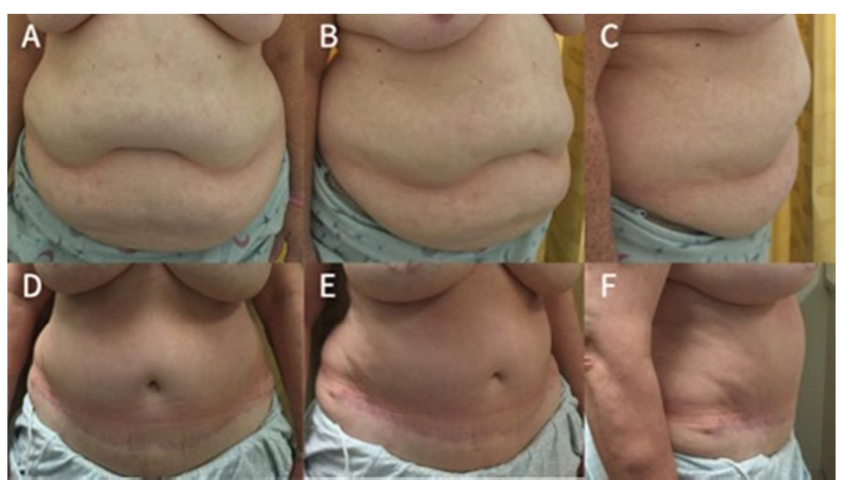

Figure 5. Patient 2 pre- and post-operative abdominal wall donor site. Example \#2 of abdominal wall donor site following bilateral msTRAM harvest. Closure was performed using inlay and onlay polypropylene mesh. $A-B$ : preoperative photographs. $C$-D: post-operative photographs demonstrating low incision and absence of hernia or bulge.

No hernias were observed $(0 \%)$, while 6 abdominal bulges were identified $(2.7 \%)$ as summarized in Table 4 . There were no differences in the rates of abdominal bulge after donor site closure in the DIEP compared with MSTRAM and ITRAM groups $(3.4 \%$ vs $2.4 \%, p=0.7)$. Differences in bulge rates of different mesh placement strategies were statistically significant: inlay only mesh $(16.7 \%)$, onlay mesh only $(4.5 \%)$, inlay and only mesh $(0.7 \%), p=0.0026$. Bulge rate was lower with polypropylene mesh use compared to biologic mesh, though this was not statistically significant $(2.4 \%$ vs $11.1 \%, p=0.12)$. Figures 4 and 5 demonstrate two examples of the post-operative outcomes that include a low transverse incision and lack of post-operative hernia and bulge.

Table 3. Abdominal donor site closure.

\begin{tabular}{lllll}
\hline Abdominal donor site closure & No Mesh & Onlay & Inlay & Inlay and Onlay \\
\hline Free TRAM and MS-TRAM & 0 & $11(6.7 \%)$ & $12(7.3 \%)$ & $141(86.0 \%)$ \\
Polypropylene & - & $11(100 \%)$ & $11(91.7 \%)$ & $138(97.9 \%)$ \\
Acellular Dermal Matrix & - & 0 & $1(8.3 \%)$ & $3(2.1 \%)$ \\
DIEP & $3(5.1 \%)$ & $56(94.9 \%)$ & 0 & 0 \\
Polypropylene & - & $51(91.1 \%)$ & - & - \\
Acellular Dermal Matrix & - & $5(8.9 \%)$ & - & - \\
\hline
\end{tabular}


Table 4. Hernia and bulge rates.

\begin{tabular}{|c|c|c|c|c|c|}
\hline & Total $(n=223)$ & DIEP $(n=59)$ & & Free and MS-TRAM $(n=164)$ & p-value \\
\hline Hernia & $0(0 \%)$ & $0(0 \%)$ & & $0(0 \%)$ & \\
\hline \multirow[t]{3}{*}{ Bulge } & $6(2.7 \%)$ & $2(3.4 \%)$ & & $4(2.4 \%)$ & 0.7 \\
\hline & Mesh Placement & $\begin{array}{l}\text { Inlay }(\mathrm{n}=12) \\
2(16.7 \%)\end{array}$ & $\begin{array}{l}\text { Onlay }(n=67) \\
3(4.5 \%)\end{array}$ & $\begin{array}{l}\text { Inlay and Onlay }(n=141) \\
1(0.7 \%)\end{array}$ & 0.0026 \\
\hline & Type of Mesh & $\begin{array}{l}\text { Polypropylene }(\mathrm{n}=211) \\
5(2.4 \%)\end{array}$ & & $\begin{array}{l}\operatorname{ADM}(\mathrm{n}=9) \\
1(11.1 \%)\end{array}$ & 0.12 \\
\hline
\end{tabular}

Other complications included donor site wound breakdown was found in 15 patients $(11.1 \%)$, which healed with local wound care. Seroma that required drainage was found in 6 patients $(4.4 \%)$. Wound infection was diagnosed in 5 patients $(3.7 \%)$, all of whom resolved with a course of oral antibiotics. No patients required mesh explantation during the study follow up period.

\section{Discussion}

Attention to the secondary abdominal defect following autologous breast reconstruction is gaining deserved attention as it relates to patient satisfaction and quality of life. [2, 3, 13] In an effort to provide an aesthetic outcome in parallel to cosmetic abdominoplasty, the senior author's preferred technique includes a low-incision, routine fascial plication and mesh reinforcement of the abdomen. There are few published descriptions on optimizing the abdominal donor site aesthetic outcome in the literature. [2, 6, 7] Nahabedian similarly advocates for preservation of the anterior rectus sheath, closure of the anterior rectus sheath primarily or with mesh, preservation of the rectus muscle and its innervation, and the use of fascial plication. [7] The author also advocates for inlay mesh placement when fascia cannot be approximated, and overlay when fascia is able to be primarily approximated, which is a technical difference from our preferred approach. [7] The importance of maintaining a low incision is also highlighted in the literature. Lindenblatt et al. similarly recommend a low incision placement of $5-7 \mathrm{~cm}$ above the vulvar commissure, while also plicating the rectus fascia following DIEP flap harvest. [6] Stalder et al. report on their technique of lowering the abdominal scar to $4-7 \mathrm{~cm}$ from the vulvar commissure and improving on abdominal wall contour. [2] The authors prefer to perform this in a staged fashion at 3 months following flap procedure. [2]

The majority of flaps in our present series were performed in an immediate fashion at the time of mastectomy $(61.9 \%$ vs $38.1 \%, \mathrm{p}<0.0001)$. Most abdominal flaps were msTRAM or fTRAM in this series compared to DIEP flaps $(73.5 \%$ vs $26.5 \%, p<0.0001)$. This significant difference follows recent trends of an increase rate of immediate reconstruction in the United States. [14] The higher incidence of msTRAM flaps reflects the senior author's effort to lower the abdominal incision. With a lower incision, large periumbilical perforators may not be incorporated into the flap. Instead, a number of smaller perforators can collectively be incorporated into the abdominal flap with and msTRAM harvest. In addition, msTRAM flap may avoid intramuscular perforator dissection that has the potential denervate and traumatize the rectus muscle, which has shown to result in donor site morbidity in DIEP flap harvest. [15] With the routine use of mesh, the deleterious effects of muscle and fascia harvest are mitigated. The relative paucity of fTRAM flap harvest reflect an effort to preserve both functional rectus muscle as well as anterior rectus sheath.

This patient series demonstrates routine use of mesh as seen in $100 \%$ of msTRAM and fTRAM donor site and $94.9 \%$ of DIEP flap closures. This liberal mesh use reflects the effort to support abdominal wall function following free flap harvest. Despite preservation of muscle and fascia, functional changes resulting in a weakened abdominal wall result following even DIEP flap harvest result. [10, 16, 17] The use of mesh is demonstrated to reduce the risk of abdominal hernia and bulging. [11, 18-21] An extreme example of abdominal rupture following DIEP flap harvest is discussed by Iwabu et al. [22] Authors report the patient required emergent surgery, which consisted of primary fascial repair with mesh overlay placement. [22]

This series of patients also demonstrated a significant difference in the rates of polypropylene mesh and biologic mesh use. The predominant use of synthetic mesh is follows recommendations from the existing abdominal wall reconstruction literature, which notes no structural benefit of biologic mesh in comparison to synthetic mesh, while also being less cost-effective. [23] In addition, the safety profile between types of mesh has been deemed similar in comparative studies. [18]

In regard to the complication of abdominal wall hernia and bulge, we found no patients in our series to have a true hernia $(0 \%)$, while $6(2.7 \%)$ patients displayed a post-operative abdominal wall bulge. These rates pair favorably to the wide range of hernia and bulge quoted in the literature at rates between $0-7 \%$ and $2.3-30 \%$, respectively. $[4,6,8,10]$ We found no difference between the rates of abdominal wall bulge between DIEP flap and msTRAM donor sites (3.4\% vs $2.4 \%, \mathrm{p}=0.7)$. Macadam et al. also found no significant differences between hernia and bulge complications between free abdominal flaps. [5] In addition, the authors noted that patient satisfaction and abdominal well-being did not differ between free abdominal flaps. [5] Cleveland et al. demonstrate similarly low rate of hernia or bulge with msTRAM and DIEP flaps with significant difference $(3.0 \%$ vs $0.4 \%, p=0.02)$. [10] However, the authors' technique of abdominal wall reinforcement differs from our technique with $96.5 \%$ of msTRAM donor sites receiving inlay mesh placement. [10] This technical difference may account for 
this subtle difference. Our authors' experience demonstrates that routine mesh placement results in fewer donor site complications, even for TRAM flaps, which are historically known to result in higher abdominal morbidity.

We observed a lower rate of abdominal wall bulge when polypropylene mesh was used compared to biologic mesh $(2.4 \%$ vs $11.1 \%, p=0.12)$, though this was not statistically significant. Patel et al. similarly found no statistically significant difference in comparing biologic mesh to synthetic mesh. [18] Here, the authors noted a similar complication profile with a slightly higher rate of hernia/bulge in their synthetic mesh group compared to the biologic mesh group ( $18 \%$ vs $8.3 \%, p=0.25)$. Chatterjee et al. evaluated the cost-effectiveness of mesh use in abdominal donor site closure based on pooled bulge/hernia rates from published reports. The authors noted that mesh use, whether synthetic or biologic, was cost effective when the produce was less than $\$ 5970$ and when hernia/bulge rates were $7.25 \%$ or less. [20] Jordan et al. reported polypropylene mesh was superior to bioabsorbable mesh when placed in an inlay fashion for donor site closure. [21] The authors reported a bulge/hernia was 13.3 times more likely when bioabsorbable mesh was used compared to polypropylene $(p=0.016)$. [21] Given these finds, the authors determined bioabsorbable mesh was not recommended for this indication. [21]

Abdominal wall bulge rate was also noted to be significantly different depending on the location of mesh placement $(p=0.0026)$. The highest rate of abdominal wall bulge was seen with inlay mesh (16.7\%), followed by onlay mesh (4.5\%), and combined inlay with onlay mesh $(0.7 \%)$. The superior functional outcomes defined by lack of hernia or abdominal bulge is seen with our preferred technique of inlay and onlay mesh use in msTRAM closure. To our knowledge, the use of both inlay and only mesh for fascial reinforcement has not been reported for msTRAM closure. In the published literature, inlay mesh alone during msTRAM, fTRAM and pedicled TRAM closure is commonly used by multiple authors. $[8,10$, $13,18]$ Our preferred technique of onlay mesh placement after DIEP harvest is consistent with previous reports that also advocate mesh reinforcement. [7, 11, 18, 20]

Despite the liberal use of mesh, our series maintains a favorable donor site infection rate of $3.7 \%$. All patients responded to a course of oral antibiotics and no patients required mesh removal. Leon et al. found no significant difference between infection rates with $(6.8 \%)$ and without mesh use $(5.0 \%)$ in their comparative study. [11] Our findings add to the existing literature disproving the infectious risk associated with synthetic mesh placement in abdominal free flap closure. [11]

The presents study is retrospective in nature, which constitutes a primary limitation. Our case series demonstrates a preferred technique by the senior surgeon, which precludes a durable control group for comparison. This lack of a control is another limitation of the present study. Furthermore, findings deemed not statistically significant may have been related to an underpowered study, which could be remedied with a larger number of patients. As we continue to refine our abdominal wall closure techniques, efforts will center on patient reported outcomes using validated tools for abdominal wall well-being. [13]

\section{Conclusion}

The abdominal donor site following autologous breast reconstruction should be optimized to provide patient satisfaction and quality of life. To parallel cosmetic abdominoplasty, our authors advocate for a low incision, fascial plication and mesh reinforcement of the abdominal wall. Our preferred technique utilizes polypropylene mesh as an overlay to reinforce primary fascial closure after DIEP harvest, while using mesh in both an inlay and onlay fashion following msTRAM and fTRAM flap harvest. This technique mitigates the morbidity of the abdominal free flap harvest, which represents a major deterrent for patients when considering abdominal based reconstruction. Further studies are underway to assess abdominal closure outcomes by means of validated patient-reported outcome instruments to further refine closure technique.

\section{Financial Disclosure Statement}

The authors have nothing to disclose. No funding was received for this article.

\section{References}

[1] Atisha DM, Tessiatore KM, Rushing CN, Dayicioglu D, Pusic A, Hwang S. A National Snapshot of Patient-Reported Outcomes Comparing Types of Abdominal Flaps for Breast Reconstruction: Plast Reconstr Surg. 2019 Mar; 143 (3): 66777.

[2] Stalder MW, Accardo K, Allen RJ, Sadeghi A. Aesthetic Refinement of the Abdominal Donor Site after Autologous Breast Reconstruction: Plast Reconstr Surg. 2015 Sep; 136 (3): 455-61.

[3] Niddam J, Bosc R, Lange F, Chader H, Hersant B, Bigorie V, et al. DIEP flap for breast reconstruction: Retrospective evaluation of patient satisfaction on abdominal results. J Plast Reconstr Aesthet Surg. 2014 Jun; 67 (6): 789-96.

[4] Erdmann-Sager J, Wilkins EG, Pusic AL, Qi J, Hamill JB, Kim HM, et al. Complications and Patient-Reported Outcomes after Abdominal-Based Breast Reconstruction: Results of the Mastectomy Reconstruction Outcomes Consortium (MROC) Study. Plast Reconstr Surg. 2018 Feb; 141 (2): 271-81.

[5] Macadam SA, Zhong T, Weichman K, Papsdorf M, Lennox PA, Hazen A, et al. Quality of Life and Patient-Reported Outcomes in Breast Cancer Survivors. Plast Reconstr Surg. 2016 Mar; 137 (3): 758-71.

[6] Lindenblatt N, Gruenherz L, Farhadi J. A systematic review of donor site aesthetic and complications after deep inferior epigastric perforator flap breast reconstruction. Gland Surg. 2019 Aug; 8 (4): 389-98. 
[7] Nahabedian MY. Achieving ideal donor site aesthetics with autologous breast reconstruction. Gland Surg. 2015 Apr; 4 (2): $145-53$.

[8] Chirappapha P, Trikunagonvong N, Prapruttam D, Rongthong S, Lertsithichai P, Sukarayothin T, et al. Donor-Site Complications and Remnant of Rectus Abdominis Muscle Status after Transverse Rectus Abdominis Myocutaneous Flap Reconstruction. Plast Reconstr Surg Glob Open. 2017 Jun; 5 (6). DOI: $10.1097 /$ GOX.0000000000001387.

[9] Clemens MW, Downey S, Agullo F, Lehfeldt MR, Kind GM, Palladino $\mathrm{H}$, et al. Clinical Application of a Silk Fibroin Protein Biologic Scaffold for Abdominal Wall Fascial Reinforcement. Plast Reconstr Surg Glob Open. 2014 Dec; 2 (11). DOI: 10.1097/GOX.0000000000000217.

[10] Cleveland EC, Fischer JP, Nelson JA, Sieber B, Low DW, Kovach SJ, et al. Optimizing the Fascial Closure: An Analysis of 1261 Abdominally Based Free Flap Reconstructions. Ann Plast Surg. 2013 Sep; 71 (3): 255-60.

[11] Leon DS, Nazerali R, Lee GK. Using Mesh to Reinforce the Abdominal Wall in Abdominal Free Flaps for Breast Reconstruction: Is There a Benefit? What are the Risks? Ann Plast Surg. 2018 Mar; 1.

[12] Karagöz H, ŞahìN C, Sever C, Külahçi Y, Eren F, Cesur C, et al. Three-layer primary closure of the bipedicled TRAM flap donor site for unilateralbreast reconstruction: a 15-year experience with 124 consecutive patients. Turk J Med Sci. 2017; 47: 861-7.

[13] Stone JP, Bello RJ, Siotos C, Meyer ML, Sebai ME, Payne RM, et al. Patient-Related Risk Factors for Worsened Abdominal Well-Being after Autologous Breast Reconstruction: Plast Reconstr Surg. 2020 Mar; 145 (3): $475 \mathrm{e}-80 \mathrm{e}$.

[14] Panchal H, Matros E. Current Trends in Postmastectomy Breast Reconstruction: Plast Reconstr Surg. 2017 Nov; 140: $7 \mathrm{~S}-13 \mathrm{~S}$.

[15] Lee K-T, Park J-W, Mun G-H. Impact of Rectus Muscle Injury during Perforator Dissection on Functional Donor Morbidity after Deep Inferior Epigastric Perforator Flap Breast Reconstruction. Plast Reconstr Surg Glob Open. 2019 Oct; 7 (10). DOI: 10.1097/GOX.0000000000002484.
[16] Ingvaldsen CA, Bosse G, Mynarek GK, Berg T, Tindholdt TT, Tønseth KA. Donor-Site Morbidity After DIEAP Flap Breast Reconstruction-A 2-Year Postoperative Computed Tomography Comparison. Plast Reconstr Surg Glob Open. 2017 Jul; 5 (7). DOI: 10.1097/GOX.0000000000001405.

[17] Uda H, Tomioka YK, Sarukawa S, Sunaga A, Sugawara Y. Comparison of abdominal wall morbidity between medial and lateral row-based deep inferior epigastric perforator flap. J Plast Reconstr Aesthet Surg. 2015 Nov; 68 (11): 1550-5.

[18] Patel KM, Shuck J, Hung R, Hannan L, Nahabedian MY. Reinforcement of the Abdominal Wall following Breast Reconstruction with Abdominal Flaps: A Comparison of Synthetic and Biological Mesh. Plast Reconstr Surg. 2014 Mar; 133 (3): 700-7.

[19] Espinosa-de-los-Monteros A, Avendano-Peza H, Novitsky YW. Abdominal Closure after TRAM Flap Breast Reconstruction with Transversus Abdominis Muscle Release and Mesh. Plast Reconstr Surg Glob Open. 2016 Sep; 4 (9). DOI: 10.1097/GOX.0000000000001014.

[20] Chatterjee A, Ramkumar DB, Dawli TB, Nigriny JF, Stotland MA, Ridgway EB. The Use of Mesh versus Primary Fascial Closure of the Abdominal Donor Site When Using a Transverse Rectus Abdominis Myocutaneous Flap for Breast Reconstruction: A Cost-Utility Analysis. Plast Reconstr Surg. 2015 Mar; 135 (3): 682-9.

[21] Jordan SW, Schulz SA, Carraher AM, Cabiling DS. Comparison of Polypropylene and Bioabsorbable Mesh for Abdominal Wall Reinforcement following Microsurgical Breast Reconstruction. J Reconstr Microsurg. 2019 Jun; 35 (05): 335-40.

[22] Iwabu J, Namikawa T, Kitagawa H, Fujisawa K, Oki T, Ogawa M, et al. Spontaneous rupture of abdominal wall after breast reconstruction using deep inferior epigastric perforator flap following mastectomy for breast cancer. Surg Case Rep. 2018 Jul; 4. DOI: 10.1186/s40792-018-0491-7.

[23] Köckerling F, Alam NN, Antoniou SA, Daniels IR, Famiglietti $\mathrm{F}$, Fortelny RH, et al. What is the evidence for the use of biologic or biosynthetic meshes in abdominal wall reconstruction? Hernia. 2018; 22 (2): 249-69. 